\title{
Direct detection of the inflationary gravitational-wave background
}

\author{
Tristan L. Smith, ${ }^{1}$ Marc Kamionkowski, ${ }^{1}$ and Asantha Cooray ${ }^{1,2}$ \\ ${ }^{1}$ California Institute of Technology, Mail Code 130-33, Pasadena, California 91125, USA \\ ${ }^{2}$ Department of Physics and Astronomy, University of California, Irvine, Irvine, California 92697, USA
}

(Received 17 June 2005; published 9 January 2006)

\begin{abstract}
Inflation generically predicts a stochastic background of gravitational waves over a broad range of frequencies, from those accessible with cosmic microwave background (CMB) measurements, to those accessible directly with gravitational-wave detectors, like NASA's Big-Bang Observer (BBO) or Japan's Deci-Hertz Interferometer Gravitational-wave Observer (DECIGO), both currently under study. Here we investigate the detectability of the inflationary gravitational-wave background at BBO/DECIGO frequencies. To do so, we survey a range of slow-roll inflationary models consistent with constraints from the CMB and large-scale structure (LSS). We go beyond the usual assumption of power-law power spectra, which may break down given the 16 orders of magnitude in frequency between the CMB and direct detection, and solve instead the inflationary dynamics for four classes of inflaton potentials. Direct detection is possible in a variety of inflationary models, although probably not in any in which the gravitational-wave signal does not appear in the $\mathrm{CMB}$ polarization. However, direct detection by $\mathrm{BBO} /$ DECIGO can help discriminate between inflationary models that have the same slow-roll parameters at CMB/LSS scales.
\end{abstract}

PACS numbers: $98.80 . \mathrm{Cq}, 04.30 . \mathrm{Db}, 04.80 . \mathrm{Nn}, 98.70 . \mathrm{Vc}$

\section{INTRODUCTION}

Long a subject of theoretical speculation, inflation [1-3] has now, with the advent of precise cosmic microwave background (CMB) measurements [4-12], become an empirical science. The concordance of the measurements with the inflationary predictions of a flat Universe and a nearly scale-invariant spectrum of primordial density perturbations [13-16] is at least suggestive and warrants further tests of inflation. Among the predictions of inflation yet to be tested is a stochastic gravitational-wave background with a nearly scale-invariant spectrum [17-23]. Detection of the CMB-polarization pattern induced by inflationary gravitational waves of wavelengths comparable to the horizon has become a goal of next-generation CMB experiments [24-28]. And now, direct detection of the inflationary gravitational-wave background (IGWB) with future spaced-based gravitational-wave detectors at deciHertz frequencies has become the subject of serious study $[29,30]$.

Detection of a gravitational-wave background, at either CMB or direct-detection frequencies, would constitute a "smoking gun" for inflation. Moreover, since the amplitude of the IGWB is determined by the energy scale of inflation at the time that the relevant distance scale exited the horizon during inflation, detection would provide important information about the new ultrahigh-energy physics responsible for inflation [31,32]. Since the frequencies probed by the $\mathrm{CMB}$ and by direct detection are separated by 16 orders of magnitude, the combination of both provides a large lever arm with which the shape of the inflaton potential can be constrained.

In this paper, we survey a range of inflationary models to investigate the detectability of the IGWB with satellite experiments, like NASA's Big-Bang Observer (BBO) [29] and the Japanese Deci-Hertz Interferometer Gravitational-wave Observatory (DECIGO) [30], currently under study. We restrict our attention to slow-roll inflation models that are consistent with measurements from the $\mathrm{CMB}$ and large-scale structure. We show how measurements of the IGWB amplitude at both CMB and directdetection scales can be used to constrain the inflationary parameter space.

Previous work [33-35] on direct detection of the IGWB has taken the gravitational-wave spectrum to be a pure power law, considered chaotic inflation [36,37] or the IGWB due to a broken scale-invariant potential [38]. In this paper, we consider a wider range of inflationary models (in the spirit of Refs. [39,40]), and we solve the inflationary dynamics to go beyond the assumption of powerlaw power spectra. With this more accurate analysis, we find that for the forms of the inflaton potential considered here the direct detection of the IGWB can break degeneracies between distinct inflationary models that produce the same slow-roll parameters at $\mathrm{CMB} /$ large-scale-structure scales for broken scale-invariant potentials.

This paper is organized as follows. In Sec. II, we review the basics and relevant ingredients of inflation as well as constraints from the CMB and large-scale structure (LSS) to the inflationary observables. We also discuss the transfer function that relates the current gravitational-wave amplitude to its primordial value. In Sec. III, we discuss the sensitivities of planned space-based gravitational-wave observatories to the IGWB. In Sec. IV, we show for four different families of slow-roll inflation models the IGWB amplitude and spectral index $n_{t}$ at BBO/DECIGO scales that are allowed by current CMB/LSS constraints to the $n_{s}-r$ parameter space (where $n_{s}$ is the scalar spectral index 
and $r$ the tensor-to-scalar ratio). Section $\mathrm{V}$ compares the results of our calculations with those obtained by extrapolating the power-law power spectra inferred from CMB/ LSS measurements to BBO/DECIGO scales. In Sec. VI we discuss a family of slow-roll inflation models where the observational signature in CMB/LSS studies for two different models is the same but differs for the direct detection of the IGWB at BBO/DECIGO scales. In Sec. VII, we summarize and make some concluding remarks.

\section{INFLATIONARY DYNAMICS AND PERTURBATIONS}

\section{A. Homogeneous evolution}

Inflation occurs when the cosmological expansion accelerates; i.e., when $\ddot{a}>0$, where $a(t)$ is the scale factor, and the overdot denotes a derivative with respect to time $t$. The evolution of the scale factor is determined by the Friedmann equation,

$$
H^{2} \equiv\left(\frac{\dot{a}}{a}\right)^{2}=\frac{8 \pi}{3 M_{\mathrm{Pl}}^{2}} \rho-\frac{K}{a^{2}},
$$

the continuity equation, $\dot{\rho}+3 H(\rho+P)=0$, and an equation of state $P(\rho)$, where $H$ is the Hubble parameter, $\rho$ is the total energy density, $P$ is the pressure, $M_{\mathrm{Pl}}$ is the Planck mass, and $K$ is a constant related to the 3-space curvature. From Eq. (1) and the continuity equation follows the "acceleration" equation,

$$
\frac{\ddot{a}}{a}=-\frac{4 \pi}{3 M_{\mathrm{Pl}}^{2}}(\rho+3 P) .
$$

For an equation of state of the form $P=w \rho$, where $w$ is a constant, inflation occurs when $w<-1 / 3$.

Consider now a spatially homogeneous scalar field $\phi$, the "inflaton." It has an energy density and pressure,

$$
\begin{aligned}
& \rho=\frac{1}{2} \dot{\phi}^{2}+V(\phi), \\
& P=\frac{1}{2} \dot{\phi}^{2}-V(\phi),
\end{aligned}
$$

from which it follows that inflation occurs if $V(\phi)>\dot{\phi}^{2}$.

The equation of motion for the inflaton is given by $\ddot{\phi}+$ $3 H \dot{\phi}+V^{\prime}=0$, where the prime denotes differentiation with respect to $\phi$. We assume that inflation has been proceeding for a long time before any observable scales have exited the horizon, and so for our purposes, the energy density is dominated by the inflaton during inflation, the curvature term, $K / a^{2}$, is negligible as compared to the inflaton energy density, and the evolution of the inflaton has been attracted to the slow-roll regime (e.g., Ref. [41]). If so, the evolution of the inflaton and the scale factor are uniquely determined by $V(\phi)$. Within the slow-roll approximation, the evolution is described by the usual slow-roll parameters,

$$
\begin{gathered}
\epsilon \equiv \frac{M_{\mathrm{Pl}}^{2}}{16 \pi}\left(\frac{V^{\prime}}{V}\right)^{2}, \\
\eta \equiv \frac{M_{\mathrm{Pl}}^{2}}{8 \pi} \frac{V^{\prime \prime}}{V}, \\
\xi \equiv \frac{M_{\mathrm{Pl}}^{4}}{64 \pi^{2}} \frac{V^{\prime} V^{\prime \prime \prime}}{V^{2}},
\end{gathered}
$$

which are required to be small compared with unity for the slow-roll approximation to be valid. Toward the end of inflation, $\epsilon$ grows, and inflation ends when $\epsilon \simeq 1$. This statement can be made precise by the use of "Hubble slow-roll" parameters [41].

\section{B. Perturbations}

To leading order in the slow-roll approximation, the amplitudes of the power spectra for density perturbations (scalar "s" metric perturbations) and gravitational waves (tensor " $\mathrm{t}$ " metric perturbations) can be written (e.g., Refs. [42,43])

$$
\begin{gathered}
\left.P_{s}(k) \approx \frac{128 \pi}{3 M_{\mathrm{Pl}}^{6}} \frac{V^{3}}{V^{\prime 2}}\right|_{k=a H}, \\
\left.P_{t}(k) \approx \frac{128}{3} \frac{V}{M_{\mathrm{Pl}}^{4}}\right|_{k=a H},
\end{gathered}
$$

as a function of wave number $k$, where $V$ and $V^{\prime}$ are evaluated when the relevant scale exits the horizon during inflation. The power spectra can be expanded in power laws,

$$
\begin{gathered}
P_{s}(k) \approx P_{s}\left(k_{0}\right)\left(\frac{k}{k_{0}}\right)^{1-n_{s}+\left(\alpha_{s} / 2\right) \ln \left(k / k_{0}\right)}, \\
P_{t}(k) \approx P_{t}\left(k_{0}\right)\left(\frac{k}{k_{0}}\right)^{n_{t}+\left(\alpha_{t} / 2\right) \ln \left(k / k_{0}\right)},
\end{gathered}
$$

where $k_{0}$ is a pivot wave number at which the spectral parameters (e.g., Ref. [44]),

$$
\begin{gathered}
n_{s}(k) \simeq 1-6 \epsilon+2 \eta, \\
n_{t}(k) \simeq-2 \epsilon, \\
\alpha_{s}(k) \simeq 16 \epsilon \eta-24 \epsilon^{2}-2 \xi, \\
\alpha_{t}(k) \simeq 4 \epsilon \eta-8 \epsilon^{2},
\end{gathered}
$$

are to be evaluated. To a first approximation, the power spectra are power laws with power-law indices $n_{s}$ and $n_{t}$, although these indices may "run" slightly with $k$, with a running parametrized by $\alpha_{s}$ and $\alpha_{t}$ [45]. Finally, the tensor-to-scalar ratio is 


$$
r \equiv \frac{P_{t}(k)}{P_{s}(k)}=16 \epsilon
$$

In this paper, we will generally evaluate $P_{s}, n_{s}$, and $\alpha_{s}$ at the distance scales of the CMB and LSS, where they are measured or constrained. In the figures below, the tensor spectral index $n_{t}$ will be evaluated at the distance scale relevant for direct detection of gravitational waves.

\section{Number of $\boldsymbol{e}$ foldings}

The number of $e$ foldings of expansion between the time, determined by $k=a_{k} H_{k}$, when a comoving distance scale labeled by $k$ exited the horizon during inflation, and the end of inflation is $N(k) \equiv \ln \left(a_{\text {end }} / a_{k}\right)$, where $a_{\text {end }}$ is the scale factor at the end of inflation. This must be (e.g., Ref. [46])

$N(k)=62-\ln \frac{k}{a_{0} H_{0}}-\ln \frac{10^{16} \mathrm{GeV}}{V_{k}^{1 / 4}}+\ln \frac{V_{k}^{1 / 4}}{V_{\text {end }}^{1 / 4}}-\frac{1}{3} \ln \frac{V_{\text {end }}^{1 / 4}}{\rho_{\mathrm{rh}}^{1 / 4}}$,

where $V_{k}$ is the inflaton potential when the comoving scale $k$ crossed the inflationary horizon; $V_{\text {end }}$ is the value of the potential at the end of inflation; and $\rho_{\mathrm{rh}} \sim T_{\mathrm{rh}}^{4}$ is the energy density of the universe once radiation domination begins. Here we have assumed that the Universe was matter dominated after inflation and before reheating and that the transition between radiation and matter domination is instantaneous. In terms of the inflaton potential, the number of $e$ foldings between two field values, $\phi_{i}$ and $\phi_{f}$, is

$$
N\left(\phi_{i}, \phi_{f}\right) \approx \frac{8 \pi}{M_{\mathrm{Pl}}^{2}} \int_{\phi_{f}}^{\phi_{i}} \frac{V(\phi)}{V^{\prime}(\phi)} d \phi,
$$

where we have supposed the potential increases as the field increases so that the field rolls towards the origin. Furthermore, if the potential determines a field value at which inflation ends, we can combine this equation with Eq. (17) and $\rho_{\text {rh }}$ to identify the field value when the current Hubble volume exited the inflationary horizon.

If we use $10^{16} \mathrm{GeV}$ for all the densities in Eq. (17), we require $62 e$ folds of inflation between the time the current horizon distance exited the horizon during inflation and the end of inflation. The strength of the IGWB is proportional to the inflaton potential height [cf., Eq. (9)], and as we will see, detectability requires $V \geqslant 10^{15} \mathrm{GeV}$. We will also see that $V_{\text {end }}$ is never much smaller than $V_{k}$. Thus, the only ratio in Eq. (17) that might be large is the last. Conservatively, the reheat temperature must be $\gtrsim 1 \mathrm{MeV}$ to preserve the successes of big-bang nucleosynthesis (BBN), implying a lower limit of $N\left(a_{0} H_{0}\right) \gtrsim 47$. This lower limit is significant, as the ratio of gravitationalwave frequencies probed by the CMB/LSS (corresponding to $k \simeq 0.05 \mathrm{Mpc}^{-1}$ ) and direct detection is $\sim e^{35}$. Therefore, if inflation results in an IGWB in the ballpark of detectability by the CMB, then inflation will last long enough to ensure the production of gravitational waves on BBO/DECIGO scales (although it does not necessarily guarantee a detectable amplitude).

\section{Constraints to inflationary observables}

We would like to survey only those inflationary models that are consistent with current data. Measurements of the "inflationary observables"-i.e., the scalar and tensor power-spectrum amplitudes, spectral indices, and running - come from CMB measurements that probe scales from the current Hubble distance $\left(\sim 10^{4} \mathrm{Mpc}\right)$ to $\sim 10 \mathrm{Mpc}$ scales, galaxy surveys that constrain the matter power spectrum from $600 \mathrm{Mpc}$ down to $20 \mathrm{Mpc}$, and from the Lyman- $\alpha$ forest, which probes down to $\sim 1 \mathrm{Mpc}$ $[47,48]$. Constraints to the classical cosmological parameters (e.g., the Hubble parameter, the deceleration parameter, the baryon density, the matter density) from other measurements help limit the range of plausible values for the inflationary observables that come from CMB/LSS measurements.

The precise constraints to the inflationary observables depend in detail on the combination of observational data sets. In our discussions, we simply take as conservative ranges $P_{s}\left(k_{0}\right)=(2.45 \pm 0.23) \times 10^{-9}, \quad n_{s}=1.0 \pm 0.1$, and $\left|\alpha_{s}\right|<0.04$ at a pivot wave number $k_{0}=$ $0.05 \mathrm{Mpc}^{-1}$ [49]. We note that the CMB-only constraints

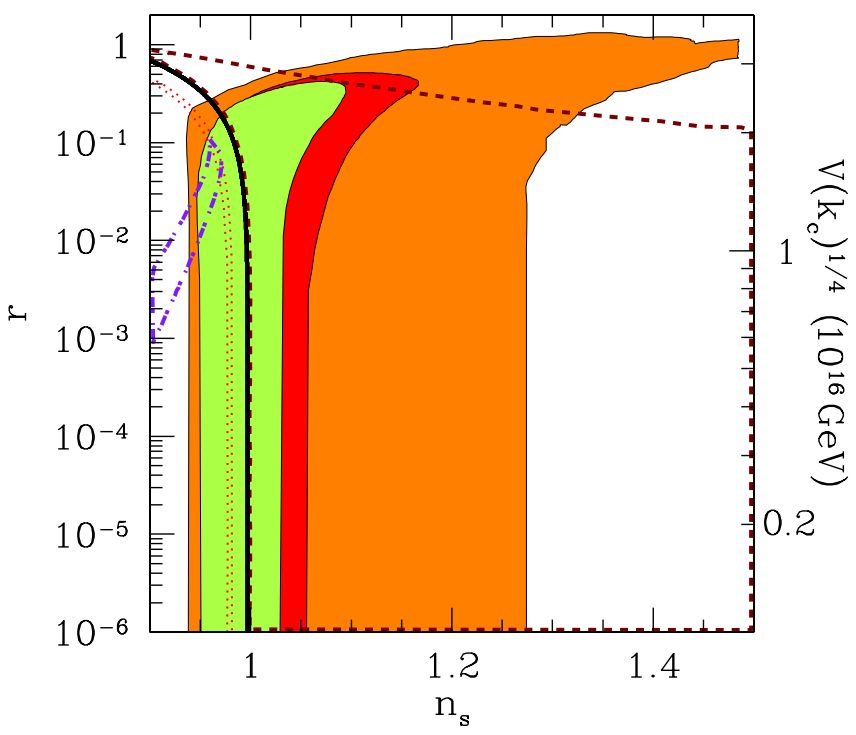

FIG. 1 (color online). Regions in the $n_{s}-r$ parameter space consistent with the CMB-only (orange/medium gray) [50], $\mathrm{CMB}$ plus galaxy surveys (red/dark gray), and CMB plus galaxy surveys plus Lyman- $\alpha$-forest constraints (green/light gray) [49]. Here, $r$ is the tensor-to-scalar ratio, and $n_{s}$ is the scalar spectral index at $\mathrm{CMB}$ scales. We plot on top of these regions the parameter space occupied by the four models of inflation we consider: power-law (solid black line), chaotic (dotted red), symmetry-breaking (dash-dotted purple) and hybrid (shortdashed dark red). The right axis shows the energy scale $\left[V\left(k_{c}\right)\right]^{1 / 4}$ of inflaton. 
correspond to a pivot wave number, $k_{0}=0.002 \mathrm{Mpc}^{-1}$ [50]. The errors we quote are not to be interpreted as statistical errors bars, except in the case of the value for $P_{s}\left(k_{0}\right)$, where the error quoted is $1 \sigma$; rather, they simply indicate a range of parameters that are in reasonable concordance with measurements [49-52] and the range of values that we use here. In our discussion, we take a conservative upper limit $r \lesssim 1$ to the tensor-to-scalar ratio. The numerical results we show in Fig. 3 use slow-roll parameters consistent with the regions in the $n_{s}-r$ parameter space, shown in Fig. 1, taken from analyses of CMB data alone, $\mathrm{CMB}$ plus galaxy surveys, and $\mathrm{CMB}$, galaxies, and the Lyman- $\alpha$ forest $[49,50]$.

\section{E. Gravitational-wave transfer function}

The gravitational-wave power spectrum $P_{t}(k)$ provides the variance $\left\langle\left|h_{k}\right|^{2}\right\rangle$ of the gravitational-wave amplitude $h_{k}$ as that mode enters the horizon. Once the wavelength is smaller than the horizon, the gravitational wave begins to oscillate, and its energy density $\rho_{k} \sim k^{2} h_{k}^{2}$ redshifts $\propto a^{-4}$ like radiation. It follows that the gravitational-wave amplitude today is $h_{k}\left(t_{0}\right)=h_{k}\left(t_{k}\right)\left(a_{k} / a_{0}\right)$, where $t_{0}$ is the time today, $t_{k}$ is the time of horizon entry, and $a_{k}=a\left(t_{k}\right)$ [53]. During radiation domination (RD), $H \propto a^{-2}$, so that $k \propto$ $1 / a_{k}$, and during matter domination (MD), $H \propto a^{-3 / 2}$, so that $k \propto 1 / a_{k}^{1 / 2}$. From these relations, we find that the value of $h_{k}$ today scales with $k$ as

$$
\begin{aligned}
& h_{k} \propto k^{-1} \text { (horizon entry during RD), } \\
& h_{k} \propto k^{-2} \text { (horizon entry during MD). }
\end{aligned}
$$

Calculations (e.g., Refs. [54,55]) of the transfer function intended for CMB predictions evolve the wave equation more carefully through matter-radiation equality, but the direct-detection frequencies are so high that the scalings we have used here are fairly precise. The sensitivities of $\mathrm{BBO} / \mathrm{DECIGO}$ will peak near a frequency $0.1 \mathrm{~Hz}$, or wave number $k=6.47 \times 10^{13} \mathrm{Mpc}^{-1}$. Matter-radiation equality corresponds to $k_{\mathrm{eq}} \approx 0.05 h^{2} \mathrm{Mpc}^{-1}$. Therefore, the primordial gravitational waves observed by the planned gravitational-wave observatories entered the horizon well before matter/radiation equality. In fact, the modes that entered the horizon during big-bang nucleosynthesis (at $T \sim 1 \mathrm{MeV}$ ) have frequencies $\sim 10^{-11} \mathrm{~Hz}$. Therefore, the gravitational waves probed by $\mathrm{BBO} / \mathrm{DECIGO}$ are $\sim 10$ orders of magnitude smaller than those associated with $\mathrm{BBN}$ and must have entered the horizon at temperatures $T \sim 10^{7} \mathrm{GeV}$.

These high temperatures imply a small correction to previous calculations, which assumed $T \propto a^{-1}$, of the transfer function due to the fact that it is actually $g_{* S}(T) a^{3} T^{3}$ that remains constant, where $g_{* S}(T)$ is the effective number of relativistic degrees of freedom contributing to the entropy density. Recalling that the Hubble parameter is $H \simeq 1.66 g_{*}^{1 / 2} T^{2} / M_{\mathrm{Pl}}$ during radiation domination (where $g_{*}$ is the effective number of relativistic degrees of freedom contributing to the energy density; at these temperatures, $g_{*} \simeq g_{* S}$ ), the condition for horizon entry for a physical wave number today $\left(a_{0} k=a_{k} H_{k}\right)$, becomes

$$
1.66 g_{*}^{1 / 2}\left(T_{k}\right)\left(T_{0} T_{k} / M_{\mathrm{PI}}\right)\left(\frac{g_{* S}\left(T_{0}\right)}{g_{* S}\left(T_{k}\right)}\right)^{1 / 3}=k .
$$

Today, $g_{* S}\left(T_{0}\right)=3.91$ for photons and three species of massless neutrinos. We then have

$$
\left(\frac{T_{k}}{3.8 \times 10^{6} \mathrm{GeV}}\right)\left[\frac{g_{*}\left(T_{k}\right)}{100}\right]^{1 / 6}=\left(\frac{k}{6.5 \times 10^{13} \mathrm{Mpc}^{-1}}\right) .
$$

Taking only standard-model particles, $g_{*}(T) \approx 100$ (or roughly double if there is low-energy supersymmetry) and should be roughly independent of temperature. We thus find that for BBO/DECIGO scales,

$$
\mathcal{T}(k)=\frac{a_{k}}{a_{0}}=2.1 \times 10^{-20}\left(\frac{k}{6.5 \times 10^{13} \mathrm{Mpc}^{-1}}\right)^{-1} g_{100}^{-1 / 6},
$$

where $g_{100} \equiv g_{*}\left(T_{k}\right) / 100$. With this transfer function, the root variance of the IGWB today is

$$
\left\langle\left|h_{k}\right|^{2}\right\rangle^{1 / 2}=P_{t}(k)^{1 / 2} \mathcal{T}(k) .
$$

Free streaming of neutrinos, which occurs after neutrinos decouple at a temperature $\sim 1 \mathrm{MeV}$, contributes an anisotropic stress [56,57]. However, the resulting damping will be negligible for modes that enter the horizon so much earlier than neutrino free streaming.

If we define the logarithmic GW contribution to the critical density,

$$
\Omega_{\mathrm{GW}}(k) \equiv \frac{1}{\rho_{c}} \frac{d \rho_{\mathrm{GW}}}{d \ln k},
$$

then [58]

$$
\Omega_{\mathrm{GW}}(k) h^{2}=\frac{c^{2} k^{2} h^{2}}{6 H_{0}^{2}}\left\langle\left|h_{k}\right|^{2}\right\rangle \equiv A_{\mathrm{GW}} P_{t}(k),
$$

where $H_{0} \equiv 100 \mathrm{~km} \mathrm{~s}^{-1} \mathrm{Mpc}^{-1}$, and $A_{\mathrm{GW}}=2.74 \times$ $10^{-6} g_{100}^{-1 / 3}$. In slow-roll inflation, $n_{t}<0$, so if we take $P_{s}\left(k_{0}\right) \simeq 2.45 \times 10^{-9}$ and $r \lessgtr 1$ (corresponding to $V^{1 / 4} \lesssim$ $\left.3.36 \times 10^{16} \mathrm{GeV}\right)$, we find an upper limit $\Omega_{\mathrm{GW}} h^{2} \lesssim$ $6.71 \times 10^{-15} g_{100}^{-1 / 3}$.

\section{DIRECT-DETECTION THRESHOLDS}

Since the detection of a stochastic background of gravitational waves has to be separated from the effect of sources of noise intrinsic to the detector, the sensitivity to a stochastic background is different than the sensitivity to a 
nonstochastic source. The total stochastic signal in a given detector can be written as a sum of a stochastic signal plus a stochastic noise, $s(t)=h_{n}(t)+h(t)$. Taking the Fourier transform of the total signal and considering the spectral density of the noise and of the stochastic signal, we find that in order to have a signal-to-noise greater than unity,

$$
\left\langle|h(f)|^{2}\right\rangle^{1 / 2} \geqslant\left(\frac{2 f S_{n}(f)}{F}\right)^{1 / 2},
$$

where $F$ is a filling factor that accounts for the fact that a primordial stochastic background will be isotropic on the sky, but the detector will only be sensitive to a fraction of the sky, while $S_{n}(f)$ is the spectral density associated with the detector noise.

For omni-directional interferometers, such as the Laser Interferometer Space Antenna (LISA), $F=2 / 5$. There is a great improvement in sensitivity when the signal from two independent detectors can be combined through a correlation analysis between the two detectors [58-61]. Such a correlation increases the sensitivity to a stochastic background such that

$\left\langle|h(f)|^{2}\right\rangle^{1 / 2} \geq 1.12 \times 10^{-2}\left(\frac{2 f S_{n}(f)}{F}\right)^{1 / 2}\left(\frac{\mathrm{Hz}}{\Delta f}\right)^{1 / 4}\left(\frac{\mathrm{yr}}{\Delta T}\right)^{1 / 4}$,

where $\Delta f$ is the bandwidth over which the signals can be correlated and $\Delta T$ is the integration time. For a correlation analysis, the increase in sensitivity is under the assumption that the detector noises are independent between the two detectors, while the only correlation expected is due to stochastic signals such as inflation. For the single detector, the minimum observable strain is independent of the integration time, while for a correlation analysis, long-term observations are essential. While LISA will not allow an opportunity for such a correlation analysis, some mission concept studies for NASA's BBO and Japan's DECIGO consider two (or more) systems such that the improvement related to the correlation analysis can be exploited. The design for LISA currently places the sensitivity at approximately $\Omega_{\mathrm{GW}} h^{2} \sim 10^{-11}$. Current designs for BBO place the sensitivity of a single detector at $\Omega_{\mathrm{GW}} h^{2} \sim 10^{-13}$ and the sensitivity of a correlated extension at $\Omega_{\mathrm{GW}} h^{2} \sim 10^{-17}$. Finally, the ultimate goal for DECIGO is a sensitivity to $\Omega_{\mathrm{GW}} h^{2} \sim 10^{-20}$ [30], corresponding to $V^{1 / 4} \sim 1.15 \times$ $10^{15} \mathrm{GeV}\left(r \sim 10^{-6}\right)$.

Besides a sensitivity to a stochastic background, one must also be concerned about sources of a stochastic background, other than inflation. One such source is the background in extragalactic supernovae [62]. Such sources have the potential to wash out any signal that would otherwise be observed from a primordial source, but the characterization of the amplitude and frequency dependence of these sources is still uncertain. Other sources of cosmological gravitational-wave backgrounds are white- dwarf/white-dwarf binaries [63], neutron-star/neutron-star binaries [64] and neutron-star/white-dwarf binaries [65].

Figure 2 shows the sensitivities to a stochastic gravitational-wave background as a function of frequency $f$ for a variety of gravitational-wave detectors. Also shown are various current limits (as solid curves) as well as a variety of projected direct and indirect sensitivities (dashed

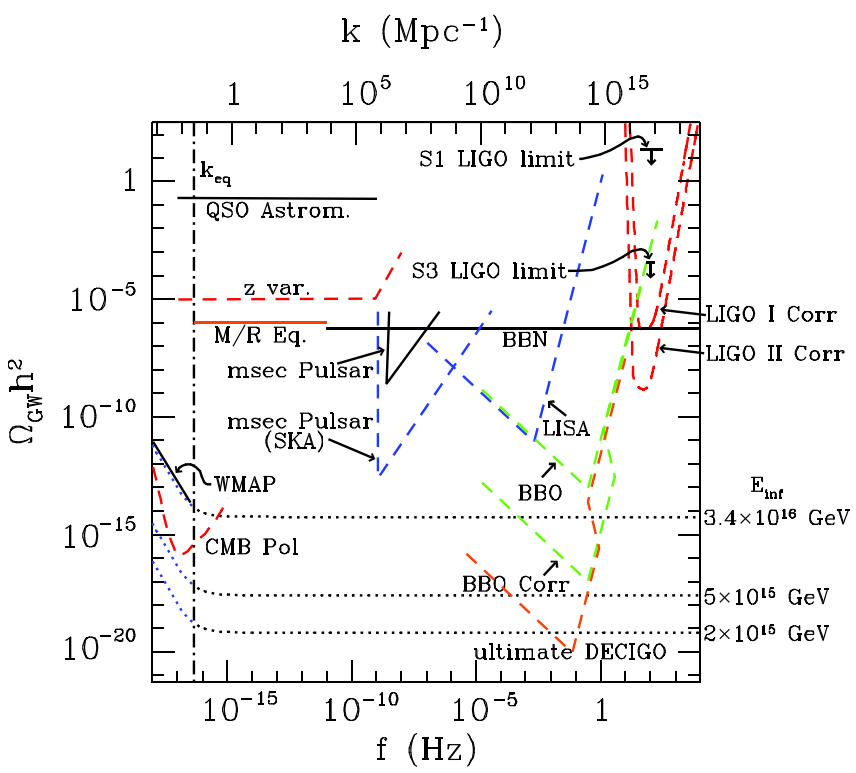

FIG. 2 (color online). Current limits and projected sensitivities to a stochastic gravitational-wave background versus the gravitational-wave frequency. The solid curves all indicate current upper limits, while the dashed curves indicate projected sensitivities. The LISA curve is from Ref. [89] and BBO correlated from Ref. [62]. The BBO sensitivity is estimated by increasing the $\mathrm{BBO}$-correlated curve by 4 orders of magnitude [see Eq. (29)]. The BBN constraint results from the limit to the number of relativistic degrees of freedom at big-bang nucleosynthesis (e.g., Ref. [90]); the "M/R" constraint is from CMB/ LSS constraints to matter-radiation equality [91]; the " $z$. var" curve is from Ref. [92]; and the quasar-astrometry limit from Refs. $[93,94]$. We note that the BBN and M/R constraints assume a scale-invariant gravitational-wave background that extends $\sim 60 e$ folds below the current Hubble horizon. LIGO sensitivities, taken from the LIGO Scientific Collaboration White Paper on Detector Research and Development [95] are given in terms of a correlated analysis between the Hanford, WA and Livingston, LA sites [see Eq. (29)]. The run 1 LIGO limit ("S1 LIGO") is from Ref. [96] and the run 3 LIGO limit ("S3 LIGO") is from Ref. [97]. Also shown are millisecond-pulsar timing constraints (current $[98,99]$ and sensitivities projected for the Square-Kilometer Array [100]). Curves corresponding to scale-invariant (i.e., $n_{t}=0$ ) gravitational-wave backgrounds are shown (dotted curves), labeled by the associated inflationary energy scales at CMB/LSS scales (but keep in mind that slowroll inflation generically predicts $n_{t}<0$, less power on small scales). The CMB/LSS currently constrains this value to be below $3.36 \times 10^{16} \mathrm{GeV}$ at CMB/LSS scales. Future CMB measurements may be able to reach energy scales near $10^{15} \mathrm{GeV}$ [101-104]. 
curves), and scale-invariant spectra parametrized by an energy scale $V^{1 / 4}$ of inflation (dotted curves). We also show limits from current CMB experiments as well as the sensitivities expected for future CMB-polarization experiments currently under study.

\section{BBO/DECIGO AMPLITUDES}

In this section, we calculate the gravitational-wave amplitude at BBO/DECIGO scales for several families of slow-roll inflation models consistent with $\mathrm{CMB} / \mathrm{LSS}$ constraints.

Measurements of the scalar amplitude $P_{s}$ and spectral index $n_{s}$ at CMB/LSS scales, as well as upper limits to the tensor contribution $r$ to the CMB and to the running $\alpha_{s}$ of the spectral index, constrain the inflaton potential and its derivatives at the field value $\phi_{c}$ that corresponds to the time at which CMB/LSS scales $k_{c}$ exited the horizon. To be precise, we use $k_{c}=0.05 \mathrm{Mpc}^{-1}$. In this work, we take as the nominal BBO/DECIGO frequency $f=0.1 \mathrm{~Hz}$, corresponding to $k=6.47 \times 10^{13} \mathrm{Mpc}^{-1}$ [and we note that $\Omega_{\mathrm{GW}}(k) h^{2} \simeq$ const for $n_{t} \simeq 0$, so our results will not depend too sensitively on the precise value of $f$ we use]. $\mathrm{CMB} / \mathrm{LSS}$ and BBO/DECIGO scales are therefore separated by $\Delta N=\ln \left(6 \times 10^{13} / 0.05\right) \simeq 35 e$ folds of inflation [66]. Equation (18) can then be used to find the field value $\phi_{g}$ at the time that BBO/DECIGO scales exited the horizon.

\section{A. Power-law inflation}

In power-law inflation, the inflaton potential takes the form,

$$
V(\phi)=V_{0} e^{-p \phi / M_{\mathrm{Pl}}} .
$$

Power-law inflation is so called because the scale factor is a power law $a(t) \propto t^{16 \pi / p^{2}}$, and the Hubble parameter also evolves as a power of time $t$. The resulting scalar and tensor power spectra are then pure power laws, with no running of the spectral indices. The parameter $\epsilon=p^{2} /(16 \pi)$ always, so that inflation must be ended artificially at some $\phi_{\text {end }}$. Although the potential has only two free parameters $\left(V_{0}\right.$ and $p$ ), there is an additional free parameter, namely, the value of $\phi_{\mathrm{c}}$, which we are free to choose in this particular family of models. This model has also $\eta=p^{2} / 8 \pi$, so $n_{s}=1-p^{2} / 8 \pi=1-2 \epsilon$, and for $n_{s}>0.9$ we find a constraint $\epsilon<0.05$. The constraint $r=16 \epsilon \lessgtr 1$ is comparable or a bit weaker. Since $n_{s}$ and $r$ depend in this model only on the parameter $p$, these models occupy a curve in the $n_{s}-r$ parameters space, which is indicated by the heavy solid curve in Fig. 1. The constraint $\Delta N=35$ to the number of $e$ folds between CMB/LSS and $\mathrm{BBO} /$ DECIGO scales tells us that

$$
\Delta N=\frac{8 \pi}{p} \frac{\phi_{g}-\phi_{c}}{M_{\mathrm{Pl}}} \simeq 35,
$$

from which it follows that

$$
\frac{P_{t}\left(k_{g}\right)}{P_{t}\left(k_{c}\right)}=\frac{V\left(\phi_{g}\right)}{V\left(\phi_{c}\right)}=e^{-\left(p^{2} / 8 \pi\right) \Delta N}=e^{-2 \epsilon \Delta N} .
$$

We thus find that the gravitational-wave amplitude at DECIGO/BBO scales is

$$
\begin{aligned}
\Omega_{\mathrm{GW}} h^{2}= & A_{\mathrm{GW}} P_{t}\left(k_{g}\right)=A_{\mathrm{GW}} r P_{s}\left(k_{c}\right) e^{-2 \epsilon \Delta N} \\
= & 1.08 \times 10^{-13} \epsilon e^{-70 \epsilon(\Delta N / 35)}\left(\frac{P_{s}\left(k_{c}\right)}{2.45 \times 10^{-9}}\right) \\
& \times\left(\frac{A_{\mathrm{GW}}}{2.74 \times 10^{-6}}\right) .
\end{aligned}
$$

This expression is maximized for $\epsilon=1 /(2 \Delta N) \simeq 1 / 70$ at a value $\Omega_{\mathrm{GW}}^{\max } h^{2}=5.68 \times 10^{-16}$. Interestingly enough, the IGWB detectability through direct detection is maximized for relatively small $\epsilon$, while the detectability with the CMB is maximized at larger $\epsilon$ [36]. Given that CMB sensitivities are expected to get to $r \sim 0.01$ in the relatively near future with the $\mathrm{CMB}$, and then to $r \sim 10^{-4}$ with a next-generation satellite experiment, it is unlikely that this model would produce a direct detection without producing a detectable CMB signal.

Figure 3(a) shows the region of the $\Omega_{\mathrm{GW}} h^{2}-n_{t}$ parameter space (at BBO/DECIGO scales) that the $n_{s}-r$ parameter space shown in Fig. 1 maps to for power-law inflation. The breadth in $\Omega_{\mathrm{GW}} h^{2}$ of the region is due to the $30 \%$ (at $3 \sigma$ ) uncertainty in $P_{s}\left(k_{c}\right)$. If power-law inflation is the correct model of inflation, then the IGWB is directly detectable with BBO for $r \geqslant 10^{-3}$ and $r \geqslant 10^{-6}$ with DECIGO.

\section{B. Chaotic inflation}

In chaotic inflation, the inflaton potential is

$$
V(\phi)=V_{0}\left(\frac{\phi}{M_{\mathrm{Pl}}}\right)^{\alpha} \text {. }
$$

In this family of models, $\epsilon(\phi)=\left(\alpha^{2} / 16 \pi\right)\left(M_{\mathrm{Pl}} / \phi\right)^{2}$, and therefore inflation ends when $\phi=\phi_{\text {end }} \equiv \alpha M_{\mathrm{Pl}} /(4 \sqrt{\pi})$. If there are $N_{c} e$ folds of inflation between the CMB horizon exit and the end of inflation, then Eq. (18) gives us $\phi_{c}^{2}=\left(M_{\mathrm{Pl}}^{2} / 16 \pi\right)\left(4 \alpha N_{c}+\alpha^{2}\right)$. We also have $\eta(\phi)=$ $\alpha(\alpha-1)\left(M_{\mathrm{Pl}} / \phi\right)^{2} /(8 \pi)$ from which it follows that at CMB/LSS scales,

$$
n_{s}=1-2 \frac{\alpha+2}{4 N_{c}+\alpha} .
$$

Noting that $47 \lesssim N_{c} \lesssim 62$, the constraint $n_{s}>0.9$ gives us a constraint $\alpha \lesssim 4 N_{c} / 19-40 / 19$. The constraint on the tensor-to-scalar ratio, $\quad r=16 \epsilon=16 \alpha /\left(4 N_{c}+\alpha\right) \lesssim 1$, leads to a slightly less stringent limit, $\alpha \lesssim 4 N_{c} / 15$. We note that the scalar running $\alpha_{s}=-2\left(1-n_{s}\right)^{2} /(2+\alpha)$ is always within the current observational constraints since $\left|1-n_{s}\right| \lesssim 0.1$. This family of models is thus parametrized by two parameters: $47 \lesssim N_{c} \lesssim 62$ and $\alpha \lesssim 4 N_{c} / 19-$ $40 / 19$. Note that each choice of $\left(\alpha, N_{c}\right)$ maps onto a point 



FIG. 3 (color online). Regions in the $\Omega_{\mathrm{GW}} h^{2}-n_{t}$ parameter space for (a) power-law, (b) chaotic, (c) symmetry-breaking, and (d) hybrid inflation. The colored (shaded) regions map out the corresponding regions in Fig. 1. Here, the gravitational-wave density $\Omega_{\mathrm{GW}} h^{2}$ and spectral index $n_{t}$ are both evaluated at DECIGO/BBO scales. Also shown are the sensitivity goals of BBO and DECIGO.

in the $n_{s}-r$ parameter space, so we could just as well choose $n_{s}$ and $r$ as our two independent parameters. If we choose to do so, then we assign $N_{c}$ and $\alpha$ by $N_{c}=(1-\epsilon) /(1-$ $\left.n_{s}-2 \epsilon\right)$ and $\alpha=4 \epsilon /\left(1-n_{s}-2 \epsilon\right)$, where $\epsilon=r / 16$.

For a fixed value of $N_{c}$, this family of models is represented by a curve in the $n_{s}-r$ parameter space; a spread in the range of values for $N_{c}$ broadens this curve into a region in the $n_{s}-r$ parameter space, as indicated by the region enclosed by the dotted red curves in Fig. 1.

Once $\alpha$ and $N_{c}$ are specified, the potential prefactor is fixed by

$$
V_{0}=\frac{3 \alpha^{2} P_{s}\left(k_{c}\right)}{128 \pi}\left(\frac{16 \pi}{4 \alpha N_{c}+\alpha^{2}}\right)^{(\alpha+2) / 2} M_{\mathrm{Pl}}^{4}
$$

The gravitational-wave amplitude at direct-detection scales is then simply

$$
\Omega_{\mathrm{GW}} h^{2}=\frac{128}{3} A_{\mathrm{GW}} \frac{V_{0}}{M_{\mathrm{Pl}}^{4}}\left(\frac{\phi_{g}}{M_{\mathrm{Pl}}}\right)^{\alpha},
$$

where the field value $\phi_{g}$ at the time direct-detection scales undergo horizon crossing is given by

$$
\phi_{g}^{2}=\frac{M_{\mathrm{Pl}}^{2}}{16 \pi}\left(4 \alpha N_{g}+\alpha^{2}\right)
$$

and where $N_{g}=N_{c}-35 \equiv N_{c}-\Delta N$ is the number of $e$ folds before the end of inflation that DECIGO/BBO scales exit the horizon. For chaotic inflation, the value of $n_{t}$ at DECIGO/BBO scales will differ from (and generally 
be larger in amplitude, or more negative than) that at CMB/ LSS scales. The value of $n_{t}$ at DECIGO/BBO scales will differ from that at CMB/LSS scales; it will be given by $n_{t}\left(\phi_{g}\right)=-2 \epsilon\left(\phi_{g}\right)$.

Figure 3(b) shows the region of the $\Omega_{\mathrm{GW}} h^{2}-n_{t}$ parameter space (at BBO/DECIGO scales) that the $n_{s}-r$ parameter space shown in Fig. 1 maps to for chaotic inflation. The breadth in $\Omega_{\mathrm{GW}} h^{2}$ of the region is due to the spread in the $\alpha-N_{c}$ parameter space for fixed $P_{s}\left(k_{c}\right)=2.45 \times 10^{-9}$; there will be a slight additional vertical broadening beyond that shown due to the uncertainty in this parameter. If chaotic inflation is the correct model of inflation, then the IGWB is directly detectable with BBO for $r \geq 10^{-3}$ and $r \gtrsim 10^{-6}$ with DECIGO.

\section{Symmetry-breaking inflation}

We now consider the Higgs potential,

$$
V(\phi)=V_{0}\left[1-\left(\frac{\phi}{\nu}\right)^{2}\right]^{2},
$$

parametrized by $V_{0}$ and a Higgs vacuum expectation value $\nu$. Our treatment of this family of models will parallel that for chaotic inflation. In these models, $\phi$ starts near the origin and then rolls toward $\phi=\nu$. The slow-roll parameters are $\epsilon(\phi)=\left(M_{\mathrm{Pl}}^{2} \phi^{2} / 4 \pi \nu^{4}\right)\left[1-(\phi / \nu)^{2}\right]^{-2}, \quad$ and $\eta(\phi)=\left(M_{\mathrm{Pl}}^{2} / 2 \pi \nu^{2}\right)\left[3(\phi / \nu)^{2}-1\right]\left[1-(\phi / \nu)^{2}\right]^{-2}$, from which we infer an end to inflation,

$$
\phi_{\text {end }}=\left[\frac{M_{\mathrm{Pl}}^{2}}{2 \pi}\left(1+2 \pi \frac{\nu^{2}}{M_{\mathrm{Pl}}^{2}}-\sqrt{1+4 \pi \frac{\nu^{2}}{M_{\mathrm{Pl}}^{2}}}\right)\right]^{1 / 2} .
$$

The field value at which CMB/LSS scales undergo horizon crossing during inflation is

$$
\phi_{c}^{2}=\frac{N_{c} M_{\mathrm{Pl}}^{2}}{\pi}+\phi_{\mathrm{end}}^{2}-2 \nu^{2} \ln \left(\phi_{\mathrm{end}} / \phi_{c}\right) .
$$

At CMB/LSS scales,

$$
n_{s}=1-\frac{1}{\pi}\left(\frac{M_{\mathrm{Pl}}}{\nu}\right)^{2} \frac{1+3 y_{c}^{2}}{\left(1-y_{c}^{2}\right)^{2}},
$$

where $y_{c} \equiv \phi_{c} / \nu$. Since $n_{s}$ is a decreasing function of $y$, the constraint $n_{s}>0.9$ requires $\nu \gtrsim 1.8 M_{\mathrm{Pl}}$. The prefactor $V_{0}$ is then fixed by the constraint,

$$
V_{0}=\frac{3}{8 \pi} P_{s}\left(k_{c}\right)\left(M_{\mathrm{Pl}} / \nu\right)^{2} \frac{y_{c}^{2}}{\left(1-y_{c}^{2}\right)^{4}} M_{\mathrm{Pl}}^{4} .
$$

Once this normalization is fixed, these models are parametrized by $\nu$ and $N_{c}$, and $n_{s}$ and $r$ are fixed once these two parameters are specified. As in chaotic inflation, we may alternatively take as our two free parameters $n_{s}$ and $r$, and then determine $\nu$ and $N_{c}$, although the inversion is not as tractable algebraically as in chaotic inflation.

The gravitational-wave amplitude at direct-detection scales is then simply

$$
\Omega_{\mathrm{GW}} h^{2}=\frac{128}{3} A_{\mathrm{GW}} \frac{V_{0}}{M_{\mathrm{Pl}}^{4}}\left(1-y_{g}^{2}\right)^{2},
$$

where $y_{g} \equiv \phi_{g} / \nu$, and the field value $\phi_{g}$ at the time directdetection scales undergo horizon crossing is given by

$$
\phi_{g}^{2}=\frac{N_{g} M_{\mathrm{Pl}}^{2}}{\pi}+\phi_{\text {end }}^{2}-2 \nu^{2} \ln \left(\phi_{\text {end }} / \phi_{g}\right),
$$

and where again $N_{g}=N_{c}-35 \equiv N_{c}-\Delta N$ is the number of $e$ folds before the end of inflation and the time when DECIGO/BBO scales exit the horizon. The value of $n_{t}$ at DECIGO/BBO scales will differ from that at CMB/LSS scales; it will be given by $n_{t}\left(\phi_{g}\right)=-2 \epsilon\left(\phi_{g}\right)$. We also note that the running of the scalar spectral index at CMB/LSS scales is

$$
\alpha_{s}=-\frac{1}{\pi^{2}}\left(\frac{M_{\mathrm{Pl}}}{\nu}\right)^{4} y_{c}^{2} \frac{5+3 y_{c}^{2}}{\left(1-y_{c}^{2}\right)^{4}} .
$$

We check in our numerical results that all of the models we consider are consistent with the bound to this parameter, $\left|\alpha_{s}\right|<0.04$. In particular we find that $\left|\alpha_{s}\right| \lesssim 10^{-3}$.

Figure 3(c) shows the region of the $\Omega_{\mathrm{GW}} h^{2}-n_{t}$ parameter space (at $\mathrm{BBO} / \mathrm{DECIGO}$ scales) that the $n_{s}-r$ parameter space shown in Fig. 1 maps to for symmetry-breaking inflation. The breadth in $\Omega_{\mathrm{GW}} h^{2}$ of the region is due to the spread in the $\nu-N_{c}$ parameter space for fixed $P_{s}\left(k_{c}\right)=$ $2.45 \times 10^{-9}$. If symmetry-breaking inflation is the correct model of inflation, then the IGWB will be detectable with BBO and DECIGO. Incidentally, we have also investigated potentials of the form $V(\phi)=V_{0}\left[1-(\phi / \nu)^{p}\right]^{2}$ with $p>$ 2 [67]. In these models, the symmetry-breaking scale can be reduced below $M_{\mathrm{Pl}}$, however the IGWB amplitude is then reduced below the level accessible to BBO/DECIGO for $\nu \lesssim 0.1 M_{\mathrm{Pl}}$.

\section{Hybrid inflation}

Hybrid inflation generally requires two scalar fields [68], but the phenomenology can be modeled by a single scalar field with the potential,

$$
V(\phi)=V_{0}\left[1+\left(\frac{\phi}{\mu}\right)^{2}\right],
$$

and the selection of a value for $\phi_{\text {end }}$ (with the only requirement that $\phi_{\text {end }}>0$ ). We note that this form for the potential is not to be taken to be generic within the class of hybrid inflation but only as a particular example. Other forms exist, such as those found in, e.g., Refs. [69-71]. Defining $y \equiv \phi / \mu$, we find that the slow-roll parameters are given by $\epsilon(y)=\left(M_{\mathrm{Pl}} / 4 \pi \mu^{2}\right) y^{2}\left[1+y^{2}\right]^{-2}$, and $\eta(y)=$ $\left(M_{\mathrm{Pl}} / 4 \pi \mu^{2}\right)\left[1+y^{2}\right]^{-1}$. In these models, $\epsilon$ is maximized at $y=1$ with a value less than unity if $\mu>M_{\mathrm{Pl}} /(4 \sqrt{\pi})$. For smaller values of $\mu$, inflation ends soon after $y=1$ $[72,73]$. The dynamics of these models resembles those of chaotic inflation, which we have already considered, and so we consider them no further. New inflationary dynamics 
arises for $\mu>M_{\mathrm{Pl}} /(4 \sqrt{\pi})$ and $y \leq 1$, and so we restrict our attention here to this regime.

The field value at which CMB/LSS scales undergo horizon crossing during inflation is

$$
y_{c}^{2}=2 \ln \left(\frac{y_{c}}{y_{\text {end }}}\right)-y_{\text {end }}^{2}-\frac{N_{c}}{2 \pi}\left(\frac{M_{\mathrm{Pl}}}{\mu}\right)^{2} .
$$

Since $y_{c}$ is taken to be a free parameter, Eq. (47), along with $47 \lesssim N_{c} \lesssim 62$, determines the value of $y_{\text {end }}$. From the slow-roll parameters,

$$
n_{s}=1+\frac{M_{\mathrm{Pl}}^{2}}{2 \pi \mu^{2}} \frac{1-2 y_{c}^{2}}{\left(1+y_{c}^{2}\right)^{2}},
$$

at CMB/LSS scales. The above expression for $n_{s}$ is maximized at $y=0$, and at this field value becomes $n_{s}=1+$ $M_{\mathrm{Pl}}^{2} / 2 \pi \mu^{2}$, which shows that we can have $n_{s}>1$ in hybrid inflation. The prefactor $V_{0}$ is then fixed by the constraint,

$$
V_{0}=\frac{3}{32 \pi}\left(\frac{M_{\mathrm{Pl}}}{\mu}\right)^{2} P_{s}\left(k_{\mathrm{c}}\right) \frac{y_{\mathrm{c}}^{2}}{\left(1+y_{\mathrm{c}}^{2}\right)^{3}} M_{\mathrm{Pl}}^{4} .
$$

Once this normalization is fixed, these models are parametrized by $\mu$ and $y_{c}$, and $n_{s}$ and $r$ are fixed once these two parameters are specified. As in chaotic inflation, we may alternatively take as our two free parameters $n_{s}$ and $r$, and then determine $\mu$ and $y_{c}$. In particular, these can be determined from

$$
\begin{gathered}
y_{c}=\left[\frac{r}{8\left(n_{s}-1\right)+2 r}\right]^{1 / 2}, \\
\mu=2\left[\frac{2}{\pi} \frac{4\left(n_{s}-1\right)+r}{\left[8\left(n_{s}-1\right)+3 r\right]^{2}}\right]^{1 / 2} M_{\mathrm{Pl}} .
\end{gathered}
$$

The gravitational-wave amplitude at direct-detection scales is then simply given by

$$
\Omega_{\mathrm{GW}} h^{2}=\frac{128}{3} A_{\mathrm{GW}} \frac{V_{0}}{M_{\mathrm{Pl}}^{4}}\left(1+y_{g}^{2}\right),
$$

where the field value $y_{g}$ at the time direct-detection scales undergo horizon crossing is given by

$$
y_{g}^{2}=2 \ln \left(\frac{y_{g}}{y_{\text {end }}}\right)-y_{\text {end }}^{2}-\frac{N_{g}}{2 \pi}\left(\frac{M_{\mathrm{Pl}}}{\mu}\right)^{2},
$$

and where again, $N_{g}=N_{c}-35 \equiv N_{c}-\Delta N$ is the number of $e$ folds before the end of inflation and the time when DECIGO/BBO scales exit the horizon. The value of $n_{t}$ at DECIGO/BBO scales will differ from that at CMB/LSS scales; it will be given by $n_{t}\left(y_{g}\right)=-2 \epsilon\left(y_{g}\right)$. The running of the tensor spectral index,

$$
\alpha_{t}(y)=\frac{1}{4 \pi^{2}}\left(\frac{M_{\mathrm{Pl}}}{\mu}\right)^{4} \frac{y^{2}\left(1-y^{2}\right)}{\left(1+y^{2}\right)^{4}},
$$

can be positive in this class of models. Thus, for $y<1$, $\alpha_{t}>0$, and the running is positive, indicating that as $y$ evolves, the tensor spectral index becomes less negative.
As we have seen in the previous models, a non-negligible gravitational-wave amplitude at CMB/LSS scales leads to a "large" amplitude at direct-detection scales primarily due to a small, negative, tensor spectral index. We therefore expect this model to produce the largest gravitationalwave amplitude at direct-detection scales. We also note that the running of the scalar spectral index at CMB/LSS scales is

$$
\alpha_{s}=\frac{M_{\mathrm{Pl}}^{4}}{2 \pi^{2} \mu^{4}} \frac{y_{c}^{2}\left(y_{c}^{2}-2\right)}{\left(1+y_{c}^{2}\right)^{4}} .
$$

With the restriction that $y_{c} \leq 1, \alpha_{s}$ is maximized at $y=$ $\sqrt{2-\sqrt{3}}$. We note from this that if $\mu \geq 0.69 M_{\mathrm{Pl}}$ the observational bound on $\alpha_{s}$ is satisfied for all $y_{c} \leq 1$. For $\mu$ not satisfying this restriction, there will be some ranges of $y_{c}$ which are incompatible with observations. This restriction is taken into account in our numerical calculations.

Figure 3(d) shows the region of the $\Omega_{\mathrm{GW}} h^{2}-n_{t}$ parameter space (at BBO/DECIGO scales) that the $n_{s}-r$ parameter space shown in Fig. 1 maps to for symmetry-breaking inflation. The breadth in $\Omega_{\mathrm{GW}} h^{2}$ of the region is due to the spread in the $\mu-N_{c}$ parameter space for fixed $P_{s}\left(k_{c}\right)=$ $2.45 \times 10^{-9}$. If hybrid inflation is the correct model of inflation, then the IGWB may be detectable with BBO and DECIGO, but it is not guaranteed.

\section{THE (RUNNING) POWER-LAW APPROXIMATION}

During inflation, the value $\phi$ of the scalar field can change very little as the scale factor $a(t)$ grows extremely rapidly. It is therefore a feature of inflation that a vast range of distance scales can correspond to a small change in $\phi$. This motivates the power-law expansions (with a slight running of the spectral index) in Eqs. (10) and (11), which assume that the inflaton potential can be accurately approximated by its Taylor expansion (to second order) about a given inflaton value. These power-law expansions are particularly appropriate when studying the CMB and largescale structure (e.g., Refs. [35,49,50]), which involve a spread in distance scales of maybe 3 orders of magnitude.

However, BBO/DECIGO frequencies are separated from those probed by the CMB/LSS by roughly 16 orders of magnitude. The inflaton may thus traverse a significant distance, and so it is not obvious that the Taylor expansion approximation that underlies the power-law approximation (even with the running of the spectral index) will remain valid. For example, in Eqs. (12) and (13), the tensor and scalar tilt are written in terms of the first-order slow-roll parameters, while second- and higher-order corrections (e.g., Ref. [74]) may be important when extending the power spectrum over large physical scales. Similarly, one must also account for higher-order derivatives of the tilt, beyond the running considered with $\alpha_{s}$ and $\alpha_{t}$. For the 
calculation performed here, higher-order corrections are not important as $\Omega_{\mathrm{GW}} h^{2}$ was directly determined with model parameters describing the inflaton potential, rather than through the power spectrum. Assuming the Taylor approximation is valid, then measurements of $P_{s}, n_{s}$, and $r$ at CMB/LSS scales fix the parameters $P_{t}\left(k_{c}\right), n_{t}$, and $\alpha_{t}$ in Eq. (11), which can then be used to predict $P_{t}\left(k_{g}\right) \propto$ $\Omega_{\mathrm{GW}} h^{2}$, the IGWB amplitude at BBO/DECIGO scales. An approach based on the Taylor expansion was considered in Ref. [35] to estimate the GW amplitude at frequencies corresponding to direct detections. Figure 4 plots the exact IGWB amplitude obtained from the calculation in the previous section versus that obtained from the power-law approximation. For small IGWB amplitudes, $r=16 \epsilon \rightarrow$ 0 , and so the potentials are very close to flat and the power law tends to be a good approximation, and is indeed a good approximation for the four classes of models we have considered. For power-law inflation, where the power spectra are precisely power laws, the two results are identical. For chaotic and symmetry-breaking inflation, $\epsilon$ becomes large when the IGWB amplitude becomes large, and $\epsilon$ evolves during inflation in such a way that the power-law approximation overestimates the true IGWB amplitude at direct-detection scales. The behavior of hybrid inflation is


FIG. 4 (color online). Here we plot the inflationary gravitational-wave background amplitude $\Omega_{\mathrm{GW}} h^{2}$ obtained with the exact inflationary dynamics described in Sec. IV versus the amplitudes obtained with the power-law approximations (with slow-roll parameters fixed by CMB/LSS observations) given in Sec. II B. The panels show results for (a) power-law, (b) chaotic, (c) symmetrybreaking, and (d) hybrid inflation. The regions are models taken from the $n_{s}-r$ parameter space in Fig. 1 (and shown in Fig. 3 ) and the blue dashed curves indicate equality. 
a bit more subtle. The running of the tensor spectral index is $\alpha_{t}=(r / 8)\left(n_{s}-1+r / 8\right)$ which, for $n_{s}>1-r / 8$ can be positive. The (running) power law would then suggest that $n_{t}$ will become positive at some small distance scale, which cannot be [see Eq. (13)]. The power-law approximation can then overestimate the true IGWB amplitude at $\mathrm{BBO} / \mathrm{DECIGO}$ scales. On the other hand, in hybrid inflation, unlike chaotic and symmetry-breaking inflation, $\epsilon$ can indeed decrease as inflation proceeds, and so the directdetection amplitude may also be underestimated by the power-law approximation. Both behaviors are seen in Fig. 4.

It should also be kept in mind that the tensor spectral index $n_{t}$ is most generally different at CMB scales than it is at direct-detection scales, and it is conceivably measurable at both. Determination of $n_{t}$ at both distance scales could therefore distinguish between inflationary models. For example, in power-law inflation, $n_{t}$ remains precisely constant, while it can change by roughly a factor of 2 for chaotic inflation for models with a directly detectable IGWB. Realistically, though, the tensor spectral indices are generically (although not in full generality) small, and so running of the tensor spectral index will be difficult to measure.

Finally, the four classes of models we have considered are not at all exhaustive, and another inflaton potential could yield a direct-detection IGWB amplitude different from those we have considered here and different from what extrapolation from CMB/LSS would suggest from the power-law approximation. For example, in models with broken scale invariance [75,76], the direct-detection amplitude could be considerably different.

\section{BROKEN SCALE-INVARIANT SPECTRUM}

To demonstrate that direct observations of the IGWB can distinguish between different forms of the inflaton potential, we consider as a toy model the broken scaleinvariant potential, which features a sharp change in the slope of the inflaton potential at some transition scale $[38,75,76]$. Such models have been invoked to explain, e.g., the paucity of dwarf galaxies observed around the Milky Way [77-79].

Consider a potential of the form,

$$
V(\phi)=V_{0} \begin{cases}(1+A \phi), & \phi \gtrsim 0, \\ (1+c A \phi), & \phi \lesssim 0,\end{cases}
$$

where $V_{0}$ is the overall normalization, $A$ is the slope of the potential at CMB/LSS scales and $c$ parametrizes how the slope changes after the break at $\phi=0$. We allow $\phi_{c}$ to be a free parameter, only requiring that it be before the break in the potential at $\phi=0$. This freedom supposes that field value at which inflation ends is not necessarily determined by the form of the potential in Eq. (55). In order to choose $\phi_{c}$, we place the break (i.e., $\phi=0$ ) $N_{0} e$ folds from $\phi_{c}$. A natural choice for $N_{0}$ is 10 , since we suppose that CMB/
LSS scales constrain the inflaton potential from $10^{4}$ to $1 \mathrm{Mpc}$. The normalization of the scalar power spectrum then fixes the normalization $V_{0}$ of the potential through the expression,

$$
V_{0}=\frac{3 P_{s}\left(k_{c}\right)}{128} \frac{A^{2} M_{\mathrm{Pl}}^{2}}{\left(1+A \phi_{c}\right)^{3}} M_{\mathrm{Pl}}^{4} .
$$

We then integrate Eq. (18), assuming the transition at $\phi=$ 0 has a negligible contribution, between $\phi_{c}$ and $\phi_{g}$ with $N=35$ in order to find $\phi_{g}$. We require that inflation not end before we reach $\phi_{g}$. For $V(\phi)$ as in Eq. (56), we find that inflation ends soon after the field reaches a value, $\phi_{*}=M_{\mathrm{Pl}} /(4 \sqrt{\pi})-(c A)^{-1}$. This then places a constraint on the combination $c A$,

$$
c A<\frac{4 \sqrt{\pi}}{M_{\mathrm{Pl}} \sqrt{1+4\left(N_{g}-N_{0}\right)}} .
$$

At CMB/LSS scales we find that $\eta\left(\phi_{c}\right)=0$, and

$$
\epsilon\left(\phi_{c}\right)=\frac{A^{2} M_{\mathrm{Pl}}^{2}}{4 A^{2} M_{\mathrm{Pl}}^{2} N_{0}+16 \pi} .
$$

From the above expression and Eqs. (12) and (16), we can see explicitly that $n_{s}$ and $r$ only depend on our choice of $N_{0}$ and $A$. At BBO/DECIGO scales we find

$$
\Omega_{\mathrm{GW}} h^{2}=4 A_{\mathrm{GW}} P_{s}\left(k_{c}\right) A^{2} M_{\mathrm{Pl}}^{2} \frac{\sqrt{A^{2} c^{2} M_{\mathrm{Pl}}^{2}\left(N_{0}-N_{g}\right)+4 \pi}}{\left(A^{2} M_{\mathrm{Pl}}^{2} N_{0}+4 \pi\right)^{3 / 2}},
$$

where $N_{g}$ is the number of $e$ foldings between $\phi_{c}$ and $\phi_{g}$. In order for there to be $N_{g} e$ folds between $\phi_{c}$ and $\phi_{g}$ the slope of the potential cannot be too large, requiring

$$
c A \leq \frac{2 \sqrt{\pi}}{M_{\mathrm{Pl}} \sqrt{N_{g}-N_{0}}} .
$$

Comparing this to Eq. (58), we find that this constraint is slightly less restrictive. We can see that this amplitude depends not only on $N_{0}$ and $A$ but also on $c$. Therefore, potentials that share approximately the same Taylor expansion at CMB/LSS scales, but different expansions at BBO/ DECIGO scales, will produce overlapping observations in the $\left(n_{s}, r\right)$ plane at CMB/LSS scales and different gravitational-wave amplitudes at BBO/DECIGO scales. With the constraint in Eq. (58), we find that as $c$ increases towards its maximum value (for a fixed $A$ ), the amplitude of the IGWB changes by an order of magnitude. For example, for $n_{s}=0.9$ and $r=0.27$ we have $1.0 \times$ $10^{-16} \lesssim \Omega_{\mathrm{GW}} h^{2} \lesssim 1.0 \times 10^{-15}$; for $n_{s}=0.99$ and $r=$ $3.16 \times 10^{-3}$ we have $2.1 \times 10^{-18} \leqslant \Omega_{\mathrm{GW}} h^{2} \leqslant$ $2.1 \times 10^{-17}$; and for $n_{s}=1.0$ and $r=3.18 \times 10^{-5}$ we have $2.1 \times 10^{-20} \lesssim \Omega_{\mathrm{GW}} h^{2} \lesssim 2.1 \times 10^{-19}$. 


\section{DISCUSSION}

In this paper we have calculated the gravitational-wave amplitude at direct-detection scales for four classes of inflationary potentials with parameters consistent with current constraints from the CMB and LSS. The gravitationalwave amplitude $\Omega_{\mathrm{GW}} h^{2}$ is proportional to the height $V\left(\phi_{g}\right)$ of the inflaton potential at the time that direct-detection comoving scales exit the horizon. Our current theoretical understanding does not fix $V\left(\phi_{g}\right)$; it is constrained to be $V^{1 / 4} \lesssim 3.4 \times 10^{16} \mathrm{GeV}$ from the $\mathrm{CMB}$, and it could conceivably be as low as $T \sim 1 \mathrm{MeV}$ without violating observational constraints. Moreover, detectability of the IGWB with $\mathrm{BBO}$ or DECIGO requires $V^{1 / 4} \gtrsim 10^{15} \mathrm{GeV}$, close to the upper allowed limit. It thus seems, a priori, that detectable models occupy a small region of parameter space.

That said, however, there are indeed constraints to inflationary models that come from the CMB and large-scale structure, notably constraints to the density-perturbation amplitude and spectral index. Figure 3 indicates that when we go through the exercise of writing down simple functional forms for the inflationary potentials and imposing current constraints, there are large regions of parameter space that lead to directly detectable IGWB amplitudes. In particular, for the symmetry-breaking potential, which looks perhaps like the type of Higgs potentials we might associate with grand unification, current constraints lead to a directly detectable IGWB amplitude.

The promise of detectability traces back to the fact that $\Omega_{\mathrm{GW}} h^{2} \propto V \propto\left(V^{\prime}\right)^{4 / 3}$, the last proportionality tracing back to Eqs. (8) and (9) for fixed density-perturbation amplitude $P_{s}$. Thus, if the potential is extremely flat, $V^{\prime} \rightarrow 0$, then the IGWB will be tiny. However, if the potential takes a form in which $V^{\prime} \sim V / \phi$, which seems theoretically natural, then the required density-perturbation amplitude is achieved with $V \sim 10^{(15-16)} \mathrm{GeV}$, the range that produces an accessible IGWB amplitude.

There is of course still plenty of room for inflation to be correct and for the IGWB amplitude to be well below the $\mathrm{BBO}$ or DECIGO threshold. For example, in power-law inflation and chaotic inflation, the IGWB amplitude becomes small when $n_{s} \rightarrow 1$; i.e., when scale invariance is achieved which, in these models corresponds to small $V^{\prime}$. On the other hand, a value $n_{s} \rightarrow 1$ does not, more generally, imply a small IGWB amplitude. For example, in hybrid inflation one can have $n_{s}=1$ if $y_{c}^{2}=1 / 2$ [cf., Eq. (48)], and for $\mu \gtrsim 1.8 M_{\mathrm{Pl}}$, the potential can reach values at CMB/LSS scales of $V \sim 3 \times 10^{16} \mathrm{GeV}$, which even after the decrease to BBO/DECIGO scales remains within reach of detection, as shown in Fig. 3(d).
There may of course be alternatives to inflation, such as cyclic models [80] or the pre big-bang model [81-86], that have completely different IGWB spectra. Although the cyclic model predicts a blue tensor spectrum, which might improve detectability at small scales, BBN constrains the amplitude of the gravitational-wave amplitude to be orders of magnitude below the BBO/DECIGO sensitivities [87].

Our conclusion is that direct detection of the IGWB is unlikely without detection with the CMB polarization. Still, direct detection could be extraordinarily valuable even if the IGWB is detected first in the CMB. Direct detection would provide yet another cross-check that the curl component in the CMB polarization is due to gravitational waves, as opposed to some other mechanism (e.g., vector modes, cosmic shear, or foregrounds). Since it occurs on such vastly different distance scales, direct detection can verify that it is a nearly scale-invariant spectrum of gravitational waves, as predicted by inflation, as opposed to some other phenomenon that might only produce large-wavelength gravitational waves. It would provide evidence for the continuation of inflation to distance scales well beyond those implied by the smoothness of the Universe suggested by the successes of BBN. The large lever arm provides an opportunity to discriminate between inflationary models that produce the same CMB/LSS observables. Even within the context of a given inflationary potential, the large lever arm associated with direct detection may allow a measurement of inflationary parameters that may be more precise than those accessible with the $\mathrm{CMB} / \mathrm{LSS}$ alone. For example, an uncertainty of $10 \%$ in $\epsilon$ from the CMB/LSS translates to a $\sim\left(10^{15}\right)^{0.2} \sim 1000$ uncertainty in the BBO/DECIGO amplitude. Thus, a detection alone, with no better than an order-unity amplitude uncertainty, corresponds to a measurement of $\epsilon$ to roughly 0.02 , probably better than is accessible with the CMB/LSS alone. Finally, the deci-Hertz IGWB amplitude counts the number of relativistic degrees of freedom at temperatures a bit above the electroweak symmetry-breaking scale, and may thus be used to probe for new degrees of freedom associated with supersymmetry of some other new physics at the electroweak scale [88]. The direct search for inflationary gravitational waves may thus be warranted.

\section{ACKNOWLEDGMENTS}

During the preparation of this paper, we learned of unpublished recent work, along similar lines, by Will Kinney as part of a BBO mission concept study [105]. This work was supported in part by DoE DE-FG03-92ER40701 and NASA NNG05GF69G. T.L.S. acknowledges the support of the NSF. 
[1] A. H. Guth, Phys. Rev. D 23, 347 (1981).

[2] A. Albrecht and P. J. Steinhardt, Phys. Rev. Lett. 48, 1220 (1982).

[3] A. D. Linde, Phys. Lett. 108B, 389 (1982).

[4] M. Kamionkowski and A. Kosowsky, Annu. Rev. Nucl. Part. Sci. 49, 77 (1999).

[5] P. de Bernardis et al. (Boomerang Collaboration), Nature (London) 404, 955 (2000).

[6] A. D. Miller et al., Astrophys. J. 524, L1 (1999).

[7] S. Hanany et al., Astrophys. J. 545, L5 (2000).

[8] N. W. Halverson et al., Astrophys. J. 568, 38 (2002).

[9] B. S. Mason et al., Astrophys. J. 591, 540 (2003).

[10] A. Benoit et al. (Archeops Collaboration), Astron. Astrophys. 399, L25 (2003).

[11] J. H. Goldstein et al., Astrophys. J. 599, 773 (2003).

[12] D. N. Spergel et al. (WMAP Collaboration), Astrophys. J. Suppl. Ser. 148, 175 (2003).

[13] A.H. Guth and S. Y. Pi, Phys. Rev. Lett. 49, 1110 (1982).

[14] J. M. Bardeen, P. J. Steinhardt, and M. S. Turner, Phys. Rev. D 28, 679 (1983).

[15] S. W. Hawking, Phys. Lett. 115B, 295 (1982).

[16] A. D. Linde, Phys. Lett. 116B, 335 (1982).

[17] L.F. Abbott and M.B. Wise, Nucl. Phys. B244, 541 (1984).

[18] A. Starobinskii, Sov. Astron. Lett. 11, 133 (1985).

[19] V. A. Rubakov, M. V. Sazhin, and A. V. Veryaskin, Phys. Lett. 115B, 189 (1982).

[20] R. Fabbri and M.D. Pollock, Phys. Lett. 125B, 445 (1983).

[21] A. A. Starobinsky, JETP Lett. 30, 682 (1979).

[22] V. Sahni, Phys. Rev. D 42, 453 (1990).

[23] B. Allen, Phys. Rev. D 37, 2078 (1988).

[24] M. Kamionkowski, A. Kosowsky, and A. Stebbins, Phys. Rev. D 55, 7368 (1997).

[25] M. Kamionkowski, A. Kosowsky, and A. Stebbins, Phys. Rev. Lett. 78, 2058 (1997).

[26] M. Zaldarriaga and U. Seljak, Phys. Rev. D 55, 1830 (1997).

[27] U. Seljak and M. Zaldarriaga, Phys. Rev. Lett. 78, 2054 (1997).

[28] P. Cabella and M. Kamionkowski, astro-ph/0403392.

[29] URL: universe.nasa.gov/program/bbo.html

[30] N. Seto, S. Kawamura, and T. Nakamura, Phys. Rev. Lett. 87, 221103 (2001).

[31] M. Kamionkowski and A. Kosowsky, Phys. Rev. D 57, 685 (1998).

[32] A. H. Jaffe, M. Kamionkowski, and L.-M. Wang, Phys. Rev. D 61, 083501 (2000).

[33] R. Bar-Kana, Phys. Rev. D 50, 1157 (1994).

[34] M. S. Turner, Phys. Rev. D 55, R435 (1997).

[35] C. Ungarelli, P. Corasaniti, R. A. Mercer, and A. Vecchio, Classical Quantum Gravity 22, S955 (2005).

[36] A. R. Liddle, Phys. Rev. D 49, 3805 (1994); 51, 4603(E) (1995).

[37] R.A. Battye and E.P.S. Shellard, Classical Quantum Gravity 13, A239 (1996).

[38] D. Polarski, Phys. Lett. B 458, 13 (1999).

[39] S. Dodelson, W. H. Kinney, and E. W. Kolb, Phys. Rev. D 56, 3207 (1997).

[40] W. H. Kinney, Phys. Rev. D 58, 123506 (1998).
[41] A. R. Liddle, P. Parsons, and J. D. Barrow, Phys. Rev. D 50, 7222 (1994).

[42] E. D. Stewart and D.H. Lyth, Phys. Lett. B 302, 171 (1993).

[43] J. E. Lidsey et al., Rev. Mod. Phys. 69, 373 (1997).

[44] A. R. Liddle and D. H. Lyth, Cosmological Inflation and Large Scale Structure (Cambridge University Press, Cambridge, UK, 2000), p. 400.

[45] A. Kosowsky and M. S. Turner, Phys. Rev. D 52, R1739 (1995).

[46] A. R. Liddle and D. H. Lyth, Phys. Rep. 231, 1 (1993).

[47] R. A. C. Croft, D. H. Weinberg, N. Katz, and L. Hernquist, Astrophys. J. 495, 44 (1998).

[48] R. Mandelbaum, P. McDonald, U. Seljak, and R. Cen, Mon. Not. R. Astron. Soc. 344, 776 (2003).

[49] U. Seljak et al., Phys. Rev. D 71, 103515 (2005).

[50] H. V. Peiris et al., Astrophys. J. Suppl. Ser. 148, 213 (2003).

[51] C. L. Bennett et al., Astrophys. J. Suppl. Ser. 148, 1 (2003).

[52] M. Tegmark et al. (SDSS Collaboration), Phys. Rev. D 69, 103501 (2004).

[53] This can be shown more rigorously by solving the equation of motion for $h$ during radiation domination and then comparing the oscillation amplitude at late times with the initial amplitude.

[54] M.S. Turner, M. J. White, and J. E. Lidsey, Phys. Rev. D 48, 4613 (1993).

[55] J. R. Pritchard and M. Kamionkowski, Ann. Phys. (N.Y.) 318, 2 (2005).

[56] S. Weinberg, Phys. Rev. D 69, 023503 (2004).

[57] S. Bashinsky, astro-ph/0505502.

[58] M. Maggiore, Phys. Rep. 331, 283 (2000).

[59] N. Christensen, Phys. Rev. D 46, 5250 (1992).

[60] E. E. Flanagan, Phys. Rev. D 48, 2389 (1993).

[61] N. J. Cornish and S. L. Larson, Classical Quantum Gravity 18, 3473 (2001).

[62] A. Buonanno, G. Sigl, G. G. Raffelt, H.-T. Janka, and E. Muller, Phys. Rev. D 72, 084001 (2005).

[63] A. J. Farmer and E. S. Phinney, Mon. Not. R. Astron. Soc. 346, 1197 (2003).

[64] R. Schneider, V. Ferrari, S. Matarrese, and S. F. Portegies Zwart, Mon. Not. R. Astron. Soc. 324, 797 (2001).

[65] A. R. Cooray, Mon. Not. R. Astron. Soc. 354, 25 (2004).

[66] We note that the actual expression that relates two field values corresponding to known length scales is not given by Eq. (18), which ignores, in part, the variation of $H$ during inflation. Instead, the exact expression is given by

$$
\ln \left(\frac{k_{1}}{k_{0}}\right)=\sqrt{\frac{4 \pi}{M_{\mathrm{Pl}}^{2}}} \int_{\phi_{1}}^{\phi_{0}} \frac{1-\epsilon}{\sqrt{\epsilon}} d \phi .
$$

The error in our expression is expected to be small, since we are only considering the epoch of inflation far from its end, so that we can always take $\epsilon \ll 1$, in which case the above expression becomes approximately equivalent to Eq. (18).

[67] W. H. Kinney and K. T. Mahanthappa, Phys. Rev. D 53, 5455 (1996).

[68] A. D. Linde, Phys. Rev. D 49, 748 (1994). 
[69] G. R. Dvali, Q. Shafi, and R. K. Schaefer, Phys. Rev. Lett. 73, 1886 (1994).

[70] A. D. Linde and A. Riotto, Phys. Rev. D 56, R1841 (1997).

[71] D. H. Lyth and E. D. Stewart, Phys. Rev. D 54, 7186 (1996).

[72] E. J. Copeland, A. R. Liddle, D. H. Lyth, E. D. Stewart, and D. Wands, Phys. Rev. D 49, 6410 (1994).

[73] One can numerically determine that within a fraction of an $e$ folding $y \rightarrow 0$ and therefore must pass through $y_{\text {end }}$.

[74] E. D. Stewart and D. H. Lyth, Phys. Lett. B 302, 171 (1993).

[75] A. A. Starobinsky, JETP Lett. 55, 489 (1992).

[76] J. Lesgourgues, D. Polarski, and A. A. Starobinsky, Mon. Not. R. Astron. Soc. 297, 769 (1998).

[77] M. Kamionkowski and A. R. Liddle, Phys. Rev. Lett. 84, 4525 (2000).

[78] A. R. Zentner and J. S. Bullock, Phys. Rev. D 66, 043003 (2002).

[79] A. R. Zentner and J.S. Bullock, Astrophys. J. 598, 49 (2003).

[80] P. J. Steinhardt and N. Turok, Science 296, 1436 (2002).

[81] G. Veneziano, Phys. Lett. B 265, 287 (1991).

[82] M. Gasperini and G. Veneziano, Phys. Rev. D 50, 2519 (1994).

[83] F. Vernizzi, A. Melchiorri, and R. Durrer, Phys. Rev. D 63, 063501 (2001).

[84] G. Veneziano, hep-th/0002094.

[85] R. Brustein, M. Gasperini, M. Giovannini, and G. Veneziano, Phys. Lett. B 361, 45 (1995).

[86] A. Buonanno, M. Maggiore, and C. Ungarelli, Phys. Rev. D 55, 3330 (1997).

[87] L. A. Boyle, P. J. Steinhardt, and N. Turok, Phys. Rev. D 69, 127302 (2004).
[88] N. Seto and J. Yokoyama, J. Phys. Soc. Jpn. 72, 3082 (2003).

[89] S. L. Larson, Online Sensitivity Curve Generator, located at http://www.srl.caltech.edu/ shane/sensitivity/; based on S. Larson, W. Hiscock, and R. Hellings, Phys. Rev. D 62 , 062001 (2000).

[90] B. Allen, gr-qc/9604033.

[91] T. L. Smith et al. (unpublished).

[92] N. Seto and A. Cooray, astro-ph/0502054.

[93] T. Pyne, C. R. Gwinn, M. Birkinshaw, T. M. Eubanks, and D. N. Matsakis, Astrophys. J. 465, 566 (1996).

[94] C. R. Gwinn, T. M. Eubanks, T. Pyne, M. Birkinshaw, and D. N. Matsakis, Astrophys. J. 485, 87 (1997).

[95] LIGO Science Collaboration White Paper, URL: http:// www.ligo.caltech.edu/docs/T/T990080-00.pdf

[96] B. Abbott et al. (LIGO Scientific Collaboration), Phys. Rev. D 69, 122004 (2004).

[97] B. Abbott et al. (LIGO Collaboration), astro-ph/0507254.

[98] V. M. Kaspi, J. H. Taylor, and M.F. Ryba, Astrophys. J. 428, 713 (1994).

[99] A. N. Lommen, astro-ph/0208572.

[100] M. Kramer, astro-ph/0409020.

[101] M. Kesden, A. Cooray, and M. Kamionkowski, Phys. Rev. Lett. 89, 011304 (2002).

[102] L. Knox and Y.-S. Song, Phys. Rev. Lett. 89, 011303 (2002).

[103] U. Seljak and C.M. Hirata, Phys. Rev. D 69, 043005 (2004).

[104] K. Sigurdson and A. Cooray, Phys. Rev. Lett. 95, 211303 (2005).

[105] S. Phinney et al., The Big-Bang Observer (NASA) mission concept study (2005). 